\title{
COMPORTAMENTO SOCIAL NA ESCOLA: DIFERENÇAS ENTRE GÊNERO E SÉRIES COMPORTAMENTO SOCIAL NA ESCOLA
}

\author{
Laura Fogaça Saud \\ Josiane Maria de Freitas Tonelotto
}

\section{Resumo}

O objetivo deste estudo foi avaliar o comportamento social em um grupo de escolares, e verificar as diferenças entre os mesmos tendo em vista aspectos como sintomas emocionais, problemas de conduta, hiperatividade, problemas de relacionamento com colegas e comportamento prósocial, contemplados no instrumento SDQ (Strengths Difficulties Questionnaire) que avalia desordens psiquiátricas relacionadas ao comportamento social. Participaram da pesquisa crianças com idade média de 9 anos e 6 meses, cursando a $3^{\text {a }}$ e $4^{\text {a }}$ séries do ensino fundamental de uma escola da rede particular de ensino, sendo 24 do gênero feminino e 17 do gênero masculino. Os resultados demonstraram que para comportamento pró-social não foram observadas diferenças quanto ao gênero. Com relação às médias obtidas em dificuldades sociais de acordo com a série, foram encontradas diferenças em problemas de conduta, problema de relacionamento com colegas e total de dificuldades. Para comportamento pró-social, foram observadas diferenças com relação à série, sendo que os escolares da $3^{\text {a }}$ série se auto-avaliaram como mais "hábeis socialmente", com relação aos estudantes da $4^{\text {a }}$ série.

Palavras-chave: Comportamento Social; Diferenças entre gêneros; Interação escolar; Ensino Fundamental.

\section{SOCIAL BEHAVIOUR IN SCHOOL: DIFFERENCES BETWEEN GENDERS AND GRADE}

\begin{abstract}
The purpose of this study was to evaluate the social behavior in a group of students and verify the differences between the genders, considering the aspects such as emotional symptons, problems of behavior, hyperactivity, problems of relationship with colleagues and pro-social behavior, contemplated in the SDQ (Strenghts Difficulties Questionnaire) wich evaluates psychiatric disorders related to the social behavior. It took part of the research children at the age of 9 years and 6 months, attending the $3^{\text {rd }}$ and $4^{\text {th }}$ grades of the elementary course in a private school, divided into 24 female and 17 male. The results showed us that concerning the pro-social behavior, differences were not observed. However, related to the averages of social difficulties were found differences in behavior problems; problems about relationship with colleagues and total of difficulties. According to pro-social behavior were found differences related to the grades, considering that the students from $3^{\text {rd }}$ grade evaluated themselves as more socially skillful than the students from the $4^{\text {th }}$ grade.
\end{abstract}

KeyWords: Social behavior; Differences between the genders; Pertaining to school interaction; Elementary Course.

\section{INTRODUÇ̃̃o}

$\mathbf{O}_{\mathrm{s}}$ relação, ele é concebido e gerado na troca mútua e na interação, cresce e desenvolve-se numa rede de relações sociais que embora se modifiquem ao longo da vida o acompanharão, e ele, será parte delas sempre.

Nos últimos anos tem aumentado o interesse de pesquisadores pela forma com que as pessoas se relacionam logo no início de seu desenvolvimento.
Observa-se que desde o nascimento o ser humano identifica-se com um conjunto de padrões de comportamento que lhe torna possível interagir e adaptar-se ao ambiente em que vive. Mesmo antes de ter adquirido comportamentos mais complexos, existe uma predisposição para que a vinculação afetiva e social se concretize (Carvalho \& Guimarães, 2002).

${ }^{1}$ Mestre em Psicologia Escolar pela PUC-Campinas - Instituto de Ensino Santo Antônio - IDESA.

2 Doutora em Ciência Médicas pela UNICAMP e Docente da PUCCAMP. 
Dois sistemas interacionais são destacados no processo de socialização infantil: o sistema adulto-criança e o sistema criança-criança. Por intermédio dessas interações a criança desenvolve suas habilidades inatas, adquire linguagem, informações, aprende regras de convivência e padrões de comportamentos aceitos e valorizados pelo meio em que vive. Além disso, aprende a expressar emoções e vivenciar experiências afetivas (Carvalho \& Guimarães, 2002; Ladd \& Hart, 1992; Ladd, Kochenderfer \& Coleman, 1996).

Os comportamentos sociais podem ser manifestados de forma positiva ou negativa. Manifestações positivas são chamadas comportamentos pró-sociais e incluem requisições, gratificações, presentes. Manifestações negativas são denominadas comportamentos anti-sociais que incluem agressões, censuras, ameaças, roubos.

De acordo com Ladd (1999) a forma pela qual as crianças constroem seus relacionamentos tem grande impacto sobre todo seu desenvolvimento. É na relação com seus pares que a criança aprende a dividir, a aguardar a vez e as melhores formas de respeitar e interagir com o outro (Hartup, 1996).

Pesquisas sobre as relações interpessoais na infância tiveram grande ênfase a partir de 1930, quando cientistas sociais iniciaram estudos sobre grupos de crianças e associação entre características sociais e posição ocupada no grupo. Esse interesse se manteve em alta até 1950 e durante os quinze anos seguintes não foi destacado. A partir de 1960 o tema voltou à tona com grande destaque e a partir dos anos 70 as pesquisas se intensificaram, sobretudo no que diz respeito ao papel essencial que possui o relacionamento com colegas ou iguais, no processo de socialização, na competência interpessoal e no ajustamento social em longo prazo (Ladd, 1999).

Miller-Johnson, Coie, Maumary-Gremaud e Bierman (2002) destacam que pesquisas sobre comportamentos sociais interessam tanto à área clinica quanto à do desenvolvimento. Kern (2001) justifica o interesse de educadores a partir da observação que muitos escolares não exibem habilidades e repertórios comportamentais necessários para que obtenham sucesso em situações acadêmicas e sociais na escola.

Miller-Johnson e colaboradores (2002) estudaram o comportamento social no desenvolvimento infantil e concluíram que a agressividade é menos comprometedora do comportamento social do que a rejeição dos colegas. Em contrapartida, o comportamento pró-social é menor em crianças que se apresentaram como agressivas e rejeitadas. Além disso, observaram que os meninos apresentaram maior nível de agressão e as meninas apresentaram-se como mais competentes socialmente.

Wentzel e Caldwell (1997) estudaram o comportamento social de escolares, relacionando-os com gênero. Verificaram que comportamentos anti-sociais têm maior estabilidade no tempo, quando comparados com os comportamentos pró-sociais. Meninos apresentaram maior número de comportamentos anti-sociais e as meninas maior número de comportamentos pró-sociais. Além disso, verificaram correlação significativa negativa entre agressividade e comportamentos pró-sociais.

Lewin, Davis e Hops (1999) apresentaram resultados de um estudo com meninos e meninas a respeito de preditividade do comportamento anti-social. Concluíram que para as meninas o maior preditivo de comportamento anti-social é a presença de problemas de escolaridade, enquanto para os meninos é a rejeição por parte dos colegas.

A presença de hiperatividade pode favorecer comportamentos anti-sociais e habitualmente está acompanhada de impulsividade, imaturidade e agressividade, e este conjunto de comportamentos produz impactos negativos sobre a aceitação da criança por seus pais, colegas e professores (Merrel, 2001). A maior incidência de exibição de comportamentos hiperativos no gênero masculino é apresentada em diversos estudos (BibouNakou, Kiosseoglou \& Stogiannidou, 2002; Lindsay \& Dockrell, 2000).

Conforme afirma Topczewski (1999) há uma predominância nítida de sintomas hiperativos para o gênero masculino, que chega à razão de 4:1. Embora a hiperatividade se apresente de forma semelhante em ambos os gêneros, o componente agressivo é a característica que se evidencia mais no gênero masculino. Para Rohde e Benczik (1999) a proporção de 4:1 é baseada em estudos realizados em serviços de saúde mental, afirmando que na realidade a proporção é de dois meninos para uma menina. Os resultados que privilegiam o gênero masculino baseiam-se no fato de que as meninas costumam apresentar mais sintomas com predomínio de desatenção, enquanto os meninos apresentam maiores problemas de comportamento. Assim, por incomodarem mais, os meninos são mais encaminhados para atendimento especializado.

Os comportamentos anti-sociais associados aos pro- 
blemas de desempenho acadêmico foram estudados por Mcevoy (2000) que encontrou uma correlação significativa e positiva entre ambos, concluindo que o comportamento anti-social é um fator preditivo de baixo desempenho escolar. Kashani e Orvaschel (apud Marcelli, 1998) mencionam ainda outro fator ligado aos comportamentos anti-sociais que interferem nas situações escolares: são os estados ansiosos. A ansiedade, quando presente, tanto na criança pequena quanto quando se torna mais velha, pode resultar em condições negativas de relacionamento causadas mais freqüentemente pela angústia de separação patológica, que predomina no gênero feminino.

Sintomas emocionais constituem-se em fatores que interferem na manifestação adequada dos comportamentos sociais. De acordo com o DSM-IV (1994); surgem em decorrência de fatores estressores identificáveis, trazendo prejuízos para a vida familiar, acadêmica e social. Manifestam-se sob a forma de depressão, choro, impotência, inquietação, ansiedade, retraimento social e queixas somáticas.

Segundo Grunspun (1999) os sintomas emocionais se constituem na apresentação de queixas somáticas ou sintomas físicos que incomodam a criança e a família. Eles não são explicados por causas físicas/orgânicas e a dimensão psicológica é prevalente. Nas crianças e adolescentes, as queixas mais comuns são sintomas dolorosos: dor de cabeça, dor no ouvido, dor de garganta, dor de estômago, dor de barriga, dor ao urinar, dor nas pernas, que são mais prevalentes no gênero feminino.

Avaliar o comportamento social não se constitui em tarefa simples principalmente pela complexidade relativa ao tema. No Brasil essa dificuldade é maximizada pelo fato de faltar estímulos para construção de instrumentos adequados e voltados para nossa realidade. Merecem destaques os trabalhos de Del Prette e Del Prette (1999; 2001) referentes a revisões teóricas e construção de instrumentos de medidas.

Em 1994 Robert Goodman, psiquiatra inglês, elaborou um questionário denominado Strengths Difficulties Questionnaire (SDQ), que avalia desordens psiquiátricas relacionadas ao comportamento social. Sua finalidade é medir comportamentos sociais adequados (capacidades) e não adequados (dificuldades) em crianças e adolescentes, na faixa etária de 3 a 16 anos. O primeiro estudo de validação apresentado por Goodman (1997) foi realizado com 403 crianças e adolescentes ingleses (4 a 16 anos), com e sem acompanhamento psiquiátrico. Os resultados obtidos foram comparados com os resultados do Questionário de Rutter (já padronizado) e se verificou alta correlação entre os resultados de ambos.

As vantagens na utilização do SDQ foram evidenciadas, principalmente em relação à formatação mais compacta, maior focalização das capacidades e dificuldades, melhores informações sobre dificuldades de atenção/hiperatividade, relação com colegas e comportamento pró-social. Além disso, o SDQ possibilita avaliação de pais e professores em versões semelhantes à utilizada com a criança e/ou adolescente.

O SDQ é um instrumento proposto inicialmente para avaliar desordens psiquiátricas relacionadas ao comportamento social em crianças e adolescentes. Foi padronizado e validado, suas capacidades psicométricas foram destacadas, tanto para utilização com populações de alto risco quanto de baixo risco de perturbações psiquiátricas. É um instrumento novo e tem sido traduzido para diversos idiomas e utilizado com satisfatoriedade em muitos países, com finalidade de avaliação clínica, de identificação de traços, em estudos epidemiológicos e principalmente com finalidade de pesquisa (Adams \& Snowiling, 2001; Bilanakis \& colaboradores. 2000; Cury \& Golfeto, 2003; Diário de Navarra, 2000; Division of Psychiatry-School of Community Health Sciences, 2000; Fleitlich, Cortazár \& Goodman, 2000; Garcia, Mazaira \& Goodman, 2000; Goodman, 1997; Goodman, 2001; Goodman \& colaboradores, 2000; Goodman \& Scott, 1999; Goodman, Meltzer \& Bailey, 1998; Goodman, Renfrew \& Mullick, 2000; Hepper \& Garralda, 2001; Klasen \& colaboradores 2000; Koskelainen, Sourander \& Vauras, 2001; Lindsay \& Dockrell, 2000; McMunn \& colaboradores 2001; Mathai, Anderson \& Bourne, 2002; Saud \& Tonelotto, 2002; Smedje, Broman \& Hetta, 2001; Smedje \& colaboradores, 1999; Thabet, Stretch \& Vostanis, 2000; Vostanis \& colaboradores, 2001).

A maioria dos estudos publicados com o SDQ ainda se refere à sua validação, grau de confiabilidade e fidedignidade, sendo que as publicações com dados de pesquisa, para que se efetuassem comparações mais esclarecedoras ainda estão sendo produzidos (Fleitlich, Cortazar \& Goodman, 2000).

No Brasil, publicações sobre a utilização do SDQ são ainda incipientes. Fleitlich e colaboradores (2000) apontaram para pontos positivos da utilização do "Suplemento de Impacto, que detalha há quanto tempo as dificuldades do comportamento existem, e o quanto 
interferem no cotidiano familiar e escolar, concluindo que seu uso leva a uma melhora importante do instrumento.

Cury e Golfeto (2002) utilizaram o SDQ numa pesquisa com 119 crianças (63\% meninos e $37 \%$ meninas) provenientes de uma escola pública, com média de idade igual a 8,18 e seus respectivos pais. Observaram pontuações elevadas e resultados considerados anormais para sintomas emocionais $(17,7 \%)$, hiperatividade $(16,8 \%)$ e para pontuação do total de dificuldades $(18,7 \%)$.

Diante de todas as considerações apresentadas, o objetivo principal dessa pesquisa foi explorar um instrumento novo para avaliar o comportamento social (dificuldades e capacidades), num grupo de escolares, e verificar a presença de possíveis diferenças entre gêneros e séries cursadas.

\section{MÉT0D0}

\section{Situação}

Os dados da presente pesquisa foram coletados numa escola de ensino infantil, fundamental e médio da rede particular de ensino, situada numa cidade do Vale do Paraíba, Estado de São Paulo. Freqüentam a escola aproximadamente 1.980 (mil novecentos e oitenta) alunos, distribuídos em salas de Educação Infantil, Ensino Fundamental ( $1^{\mathrm{a}}$ a $8^{\mathrm{a}}$ série) e Ensino Médio (Comum e Informática). Os alunos estão na faixa etária aproximada de dois (dois) a 18 (dezoito) anos.

\section{Participantes}

Escolares - Dos 140 alunos convidados $\left(3^{\mathrm{a}}\right.$ e $4^{\mathrm{a}}$ séries), 41 foram autorizados pelos seus responsáveis a participar, sendo 24 do gênero feminino e 17 do gênero masculino. Vinte e um participantes freqüentavam a terceira série (14 do gênero feminino e 7 do gênero masculino) e vinte participantes frequientavam a quarta série (10 do gênero masculino e 10 do feminino). Os escolares apresentaram uma média de idade de nove anos e 6 meses.

\section{Material}

Termo de Consentimento Livre e Esclarecido utilizado com a finalidade de fornecer informações a respeito dos objetivos da pesquisa e a forma de participação, além de assegurar o caráter sigiloso de tratamento das informações obtidas.
Questionário de Capacidades e Dificuldades (SDQ) (Goodman, 1999a) - O questionário apresenta três versões, indicadas para serem respondidas pelos alunos, seus pais ou responsáveis e por professores. Apresenta inicialmente instruções para preenchimento (comportamento da criança com base nos últimos seis meses) e dados identificação (iniciais e data de nascimento). As questões são objetivas, distribuídas, respectivamente, por escalas. O questionário é composto por 25 (vinte e cinco) itens contidos em 5 (cinco) escalas: Sintomas Emocionais, Problemas de Conduta, Hiperatividade, Problemas de Relacionamento com Colegas e Comportamento Pró-Social. As alternativas para resposta são expostas em cada questão, e apresentam como opções: falso, mais ou menos verdadeiro e verdadeiro.

$\mathrm{O}$ instrumento possui uma segunda parte, somente para os pais e professores, denominada Suplemento de Impacto, com cinco (cinco) questões. Nesse estudo a escala de impacto não foi utilizada por reconhecer-se que se trata de dados que interessam mais a estudos epidemiológicos ou com populações de risco, proposta pelo autor posteriormente à criação da escala, como acessório para melhor identificação do grau de dificuldades sociais.

\section{Procedimento}

\section{Coleta de dados}

A Escola foi informada da intenção de se realizar uma coleta de dados em suas dependências, e para isso foi formulada uma carta-pedido, solicitando autorização para a realização do estudo junto aos alunos, professores e pais. Após a anuência dos diretores, outra cartapedido foi encaminhada aos pais dos alunos, solicitando autorização e contendo informações e explicações sobre o objetivo da pesquisa. Após autorização dos pais, quanto ao envolvimento dos filhos na coleta de dados a mesma foi posta em andamento.

Foram incluídas na coleta de dados, crianças de duas turmas de cada série, ou seja, alunos que estavam cursando a $3^{\mathrm{a}}$ série A e B e a $4^{\mathrm{a}}$ série $\mathrm{A}$ e $\mathrm{B}$ do ensino fundamental. No total de alunos, somando-se as quatro turmas, foram distribuídas 140 cartas-convite aos pais ou responsáveis, contendo o termo de consentimento livre e esclarecido. Retornaram, ao todo, 41 autorizações desta forma. A pesquisa foi realizada tendo como participantes estes 41 alunos. 
A aplicação dos questionários dos alunos ocorreu em sessão coletiva que foi organizada e realizada pela pesquisadora. Após uma conversa informal com os participantes, foram oferecidas informações necessárias para que o questionário fosse respondido. Iniciada a aplicação e ao término dela não foram manifestadas dificuldades no preenchimento do questionário.

\section{Análise dos dados}

Os dados foram descritos e analisados por meio de provas estatísticas que oportunizaram a obtenção de frequiências, comparação de médias e análise de correlação.

As cinco escalas que compõem o instrumento, quatro de dificuldades (sintomas emocionais, problemas de conduta, hiperatividade e problemas de relacionamento com colegas) e de capacidades (comportamento prósocial) foram pontuadas ( 25 itens), e em seguida obtevese a Pontuação Total de Dificuldades conforme definido por Goodman, 1999. Para a interpretação da pontuação obtida em capacidades e dificuldades Goodman (1999) definiu três categorias: normal, limítrofe e anormal.

\section{RESULTADOS E DISCUSSÃo}

A análise das pontuações gerais, normais, limítrofes e anormais em relação às dificuldades, conforme apresentação na Figura 01, revelou que quanto aos sintomas emocionais, que apenas escolares do gênero feminino, tanto da terceira quanto da quarta série, pontuaram na indicação limítrofe.

Pode-se, desta forma, observar que as meninas demonstram uma maior tendência a introjetar as dificuldades e somatizá-las, quando comparadas suas respostas com as dos meninos. Estes dados são compatíveis com os achados na literatura a respeito de sintomas emocionais, conforme afirmações de Marcelli (1998) e Grunspun (1999).

A observação de maior pontuação para sintomas emocionais, por parte de escolares do gênero feminino pode relacionar-se à possibilidade deste fato refletir características do meio cultural do tipo de sociedade em que os participantes estão inseridos, que provavelmente permita à menina exibir maior número de queixas do que os meninos.

Não se pode desconsiderar que a aprendizagem das regras sociais pelas meninas ocorre de forma diferenciada que para os meninos. Os pais ainda mantêm algumas regras diferenciadas para educar meninos e meninas. Meninos são criados para serem fortes e desaconselha-se que demonstrem seus sentimentos. Às meninas permitem-se maiores comportamentos emotivos e maior expressão de sentimentos. Desta forma há uma propensão em se criar formas de atuação diferenciada para meninos e meninas, bem como de comportamentos diferenciados para ambos de acordo com o que afirmam Fontana (1991), Del Prette e Del Prette (1999), Trad (1999) e Bierman (2001).
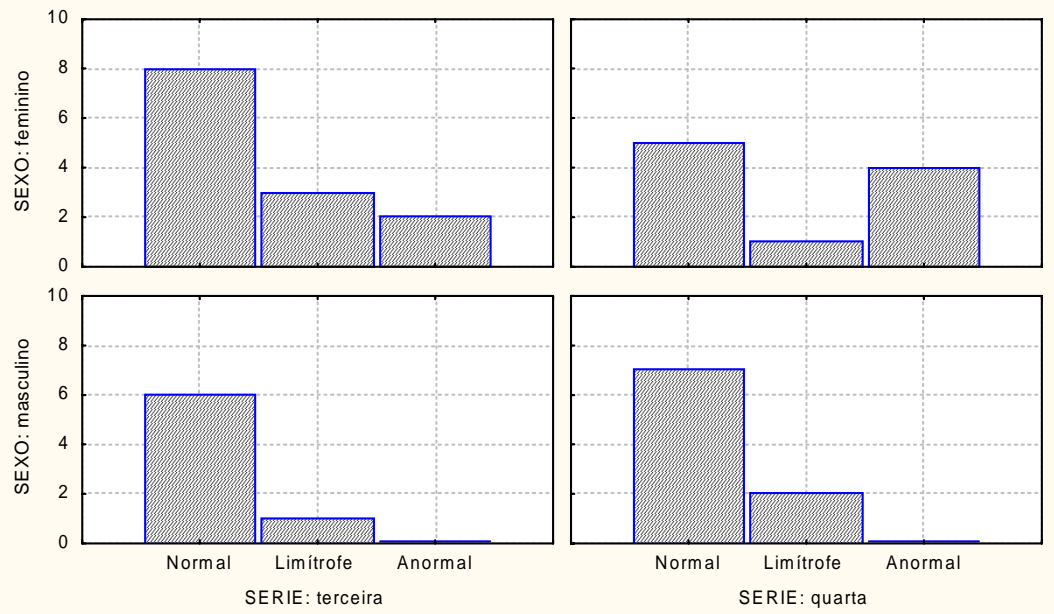

Figura 01 - Distribuição dos participantes de acordo com as pontuações normais, limítrofes e anormais em sintomas emocionais no SDQ, de acordo com gênero e com a série. 
Em relação aos resultados obtidos em problemas de comportamento ou de conduta, conforme dados da Figura 02 , os resultados na categoria normal são mais evi- externalizados e avaliados como inadequados ou mesmo agressivos que são mais característicos do gênero masculino.
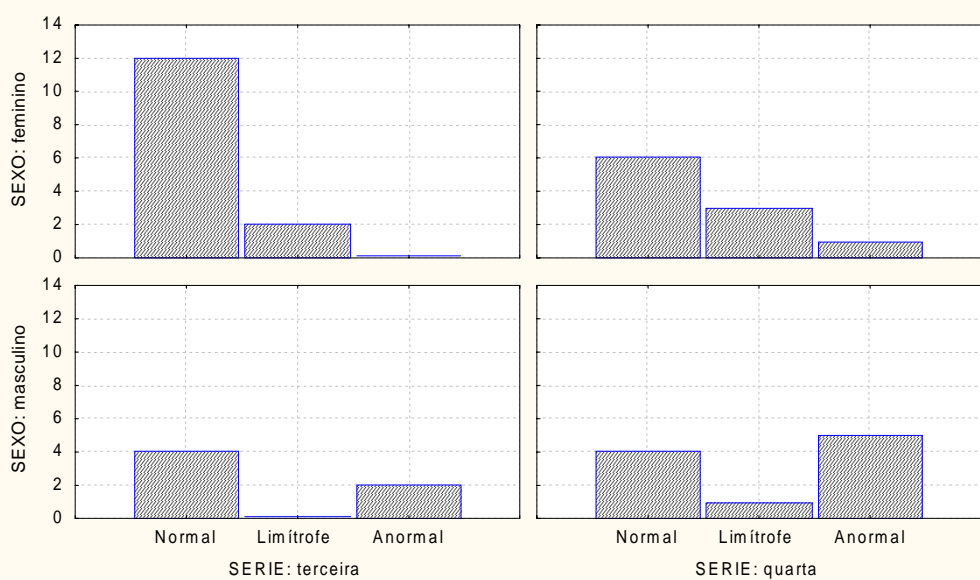

Figura 02 - Distribuição dos participantes de acordo com as pontuações normais, limítrofes e anormais em problemas de conduta no SDQ de acordo com gênero e série.

denciados por escolares do gênero feminino da terceira série, sendo que resultados anormais são mais observados para escolares do gênero masculino da quarta série.

De acordo com os dados apresentados na Figura 02, é possível observar que enquanto as meninas da quarta série apresentaram maiores índices em sintomas emocionais, os meninos desta série apresentaram maiores índices em problemas de comportamento ou conduta. Estes achados estão de acordo com os achados relatados por Miller-Johnson e colaboradores (2002) e Wentzel e Caldwell (1997) quanto aos comportamentos
Neste aspecto também é possível observar a influência da cultura e da sociedade na forma diferenciada de educar meninos e meninas, permitindo número maior de comportamentos agressivos aos meninos, o que pode ser determinante para a observação das diferenças (Bierman 2001; Del Prette \& Del Prette, 1999; Fontana, 1991; Tradd, 1999).

Em hiperatividade, as pontuações normais foram mais freqüentes para todos os escolares, mas observaram-se alguns dados que revelam a ocorrência de pontuações normais mais freqüentes em escolares
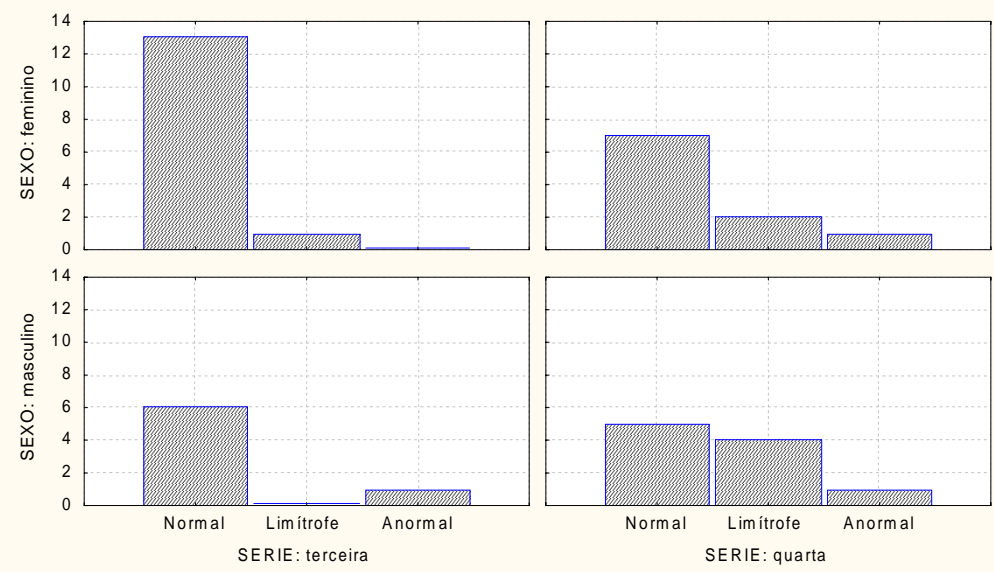

Figura 03 - Distribuição dos participantes de acordo com as pontuações normais, limítrofes e anormais em hiperatividade no SDQ de acordo com gênero e série. 
do gênero feminino da terceira série, e quanto à pontuação anormal as freqüências são semelhantes para escolares do gênero masculino da terceira e quarta séries, e para o gênero feminino da quarta série.

Os resultados apresentados na Figura 03 apontam para uma tendência, por parte dos meninos, a exibir mais comportamentos ditos "inadequados" socialmente do que as meninas, mas as meninas da quarta série foram as que também pontuaram mais, confirmando que podem estar vivendo uma fase que está favorecendo o aparecimento de sintomas. É preciso considerar que fatores opinião de Topczewski (1999), Rohde e Benczik (1999), Dumas e Pelletier (1999) e Lindsay e Dockrell (2000).

Em problemas de relacionamento com colegas, também se constatou que as pontuações normais foram mais freqüentes para todos os escolares, porém foi possível perceber que um maior número de resultados normais foi detectado em escolares da terceira série com predomínio do gênero feminino, e que resultados anormais estiveram mais presentes em escolares da quarta série, de ambos os gêneros. Novamente o grupo de escolares da quarta série foi o que apresentou maior número de pontuações anormais.
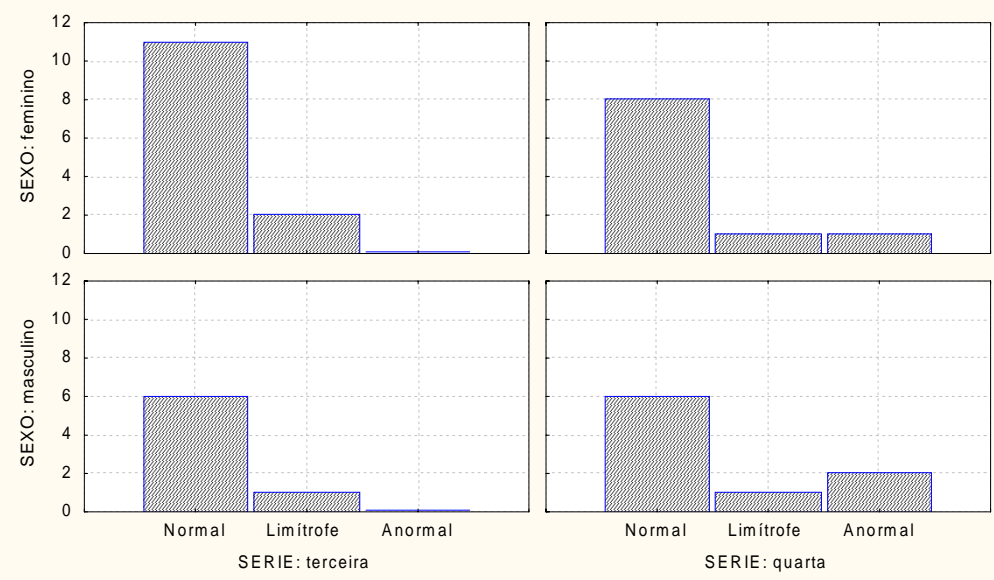

Figura 04 - Distribuição dos participantes de acordo com as pontuações normais, limítrofes e anormais em problemas de relacionamento com colegas no SDQ de acordo com gênero e série.

como a ansiedade e a expectativa frente à próxima etapa escolar, a $5^{\text {a }}$ série, possam ser determinantes para os resultados encontrados. A hiperatividade é uma característica essencialmente masculina conforme a
Na Figura 04 é apresentada uma análise das pontuações gerais, normais, limítrofes e anormais em relação às capacidades no que se refere ao comportamento prósocial. Observa-se que as pontuações normais foram

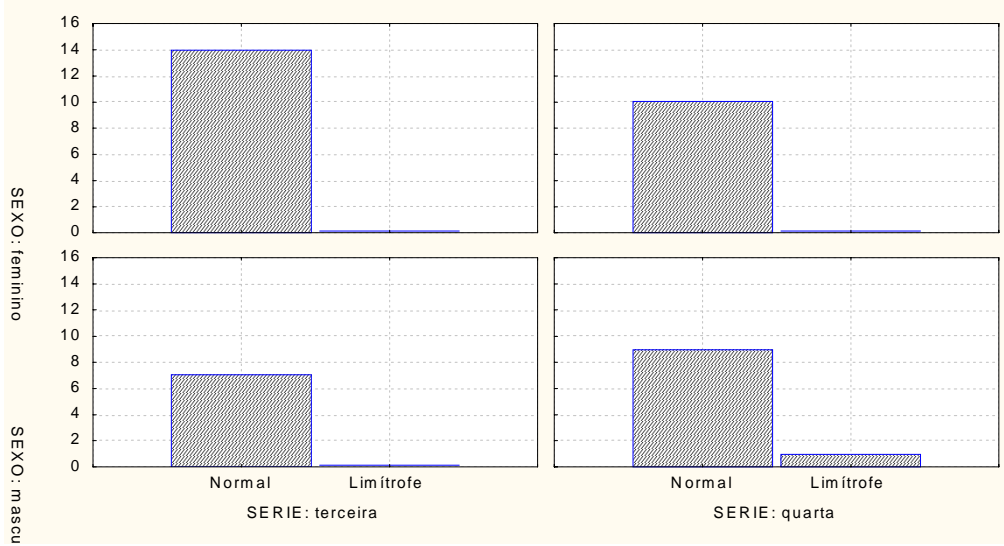

Figura 05 - Distribuição dos participantes de acordo com as pontuações normais, limítrofes e anormais em comportamento pró-social no SDQ de acordo com gênero e série. 
registradas para a maioria dos escolares independentemente de gênero e de série, apesar de ter-se detectado que a maior quantidade de resultados normais foram obtidos por escolares da terceira série.

Dados da Tabela 01 permitem que sejam comparadas as médias obtidas pelos escolares em comportamentos sociais de acordo com o gênero. Diferenças foram encontradas em sintomas emocionais com médias superiores para o gênero feminino. Além disto, são apresentados dados referentes aos comportamentos sociais de acordo com a série. Observaram-se diferenças entre problemas de comportamento ou conduta, problemas de relacionamento com os colegas, total de dificuldades e comportamento pró-social. as meninas se apresentaram como mais competentes socialmente. Além disso, no estudo de Adams e colaboradores (1999) verificou-se que as meninas apresentaram maior número de problemas no relacionamento com os colegas, fato não observado no presente estudo.

De forma geral os participantes fizeram uma autoavaliação positiva em relação ao comportamento prósocial. De 41 participantes de terceira e quarta série do ensino fundamental, 17 eram do gênero masculino e 24 do gênero feminino, detectou-se uma média 9,0 para o gênero feminino e 8,11 para o gênero masculino, sendo que a $3^{\text {a }}$ série obteve média 9,42 e a $4^{\text {a }}$ série uma média 7,8 , conforme mostrou a tabela 4 , com diferença significativa entre médias em relação à série, o que vale

Tabela 01 - Comparação das médias obtidas pelos participantes de acordo com o gênero e de acordo com a série em dificuldades sociais no SDQ, utilizando-se o teste t de student.

\begin{tabular}{|c|c|c|c|c|c|c|}
\hline & \multicolumn{2}{|c|}{ Gênero } & \multirow[t]{2}{*}{ Análise estatística } & \multicolumn{2}{|c|}{ Série } & \multirow[t]{2}{*}{ Análise estatística } \\
\hline & FEM & MAS & & $3^{\mathrm{a}}$. & $4^{\mathrm{a}}$. & \\
\hline $\begin{array}{l}\text { Sintomas } \\
\text { emocionais }\end{array}$ & 4,56 & 2,81 & $\begin{array}{c}\mathrm{t}=2,4 \\
\mathrm{p}=0,021 * \\
\mathrm{p}<0,05\end{array}$ & 3,5 & 4,21 & $\begin{array}{c}\mathrm{t}=-0,930 \\
\mathrm{p}=0,35\end{array}$ \\
\hline $\begin{array}{l}\text { Problemas de } \\
\text { conduta }\end{array}$ & 2,37 & 3,37 & $\begin{array}{l}\mathrm{t}=-1,44 \\
\mathrm{p}=0,157\end{array}$ & 1,9 & 3,65 & $\begin{array}{c}\mathrm{t}=-2,745 \\
\mathrm{p}=0,009^{*} \\
\mathrm{p}<0,05\end{array}$ \\
\hline Hiperatividade & 3,37 & 3,64 & $\begin{array}{l}t=-0,39 \\
p=0,694\end{array}$ & 2,9 & 4,1 & $\begin{array}{c}\mathrm{t}=-1,834 \\
\mathrm{p}=0,742\end{array}$ \\
\hline $\begin{array}{l}\text { Problemas de } \\
\text { relacionamento } \\
\text { com os colegas }\end{array}$ & 2,30 & 2,75 & $\begin{array}{l}t=-0,71 \\
p=0,476\end{array}$ & 1,9 & 3,1 & $\begin{array}{c}\mathrm{t}=-2,075 \\
\mathrm{p}=0,044^{*} \\
\mathrm{p}<0,05\end{array}$ \\
\hline $\begin{array}{l}\text { Total de } \\
\text { dificuldades }\end{array}$ & 13,04 & 12,07 & $\begin{array}{c}t=0,58 \\
p=0,564\end{array}$ & 10,56 & 14,66 & $\begin{array}{c}\mathrm{t}=-2,683 \\
\mathrm{p}=0,0111^{*} \\
\mathrm{p}<0,05\end{array}$ \\
\hline $\begin{array}{l}\text { Comportamento } \\
\text { Pró - social }\end{array}$ & nove & 8,11 & $\begin{array}{l}\mathrm{t}=1,834 \\
\mathrm{p}=0,074\end{array}$ & 9,42 & 7,8 & $\begin{array}{c}\mathrm{t}=3,881 \\
\mathrm{p}=0,0003^{*} \\
\mathrm{p}<0,05\end{array}$ \\
\hline
\end{tabular}

Os resultados apresentados na Tabela 1 corroboram os encontrados nos estudos de Marcelli (1998) e Grunspun (1999). Em relação ao gênero masculino, os resultados não acompanham o que traz a literatura, quando apontam para maior freqüência de hiperatividade e problemas de comportamento ou conduta para este gênero (Bibou-Nakou, Kiosseoglou \& Stogiannidou, 2001; Lindsay \& Dockrell, 2000; Miller-Johnson \& colaboradores, 2002; Rohde \& Benczik, 1999; Topczewski, 1999; Wentzel \& Caldwell, 1997).

Para comportamento pró-social não foram observadas diferenças quanto ao gênero. Esses resultados contrastam com os resultados apresentados por Adams e colaboradores (1999) e Miller-Johnson e colaboradores (2002), no quais dizer que os escolares de terceira série se auto-avaliaram como mais "hábeis" socialmente.

Neste aspecto, não podemos desconsiderar que a idade cronológica, mesmo que tendo uma diferença em linhas gerais de apenas um ano, pode influenciar no julgamento de si mesmo e na forma de perceber-se em relação ao ambiente, neste enfoque poderemos mencionar o processo de autoconceitos que se encontram de alguma forma mais elaborados, e assim, as autodescrições tornam-se mais equilibradas, e a atribuição de valores positivos ou negativos aos próprios atributos pessoais tornam-se mais consistentes, envolvendo uma auto-avaliação mais "amadurecida", conforme citado em Newcombe (1999). 
Cabe ainda, uma ressalva de extrema importância para os achados do presente estudo. Apesar da importância dos resultados encontrados e das relações com a literatura consultada, não se pode esquecer que o

\section{REFERÊNCIAS}

Adams, J.W., Snowling, M.J., Hennessy, S., \& Kind P. (1999). Problems of behavior, reading and arithmetic: assessment of comorbidity using Strengths and Difficulties Questionnaire. British Journal of Educational Psychology, 69, 571-585.

Adams, J.W., \& Snowling, M.J. (2001). Executive function and reading impairments in children reported by their teachers as "hiperactive". British Journal of Developmental Psychology, 19, 293-306.

American Psychiatric Association (1994). Diagnostic and Statistical Manual of mental disorders.( $4^{\text {th }}$ ed.) Washington. D.C: American Psychiatric Association.

Bee, H. (1997). O Ciclo Vital. Porto Alegre: Artes Médicas.

Bibou-Nakou, I., Kiosseoglou, G., \& Stogiannidou, A. (2002). Strengths and difficulties of school-aged children in the family and school context. Psychology: The Journal of the Hellenic Psychological Society, 8, 506-525.

Bilanakis, N.D., Pappas, E.E., Lecic, T., Dusika,A., \& Dimitra, B. (2000). Children of war fostered by Greek families for six months: The effect of the programme on children and foster mothers by. European Child and Adolescent Psychiatry, 13, 215-222. [Abstract].

Bierman, K.L. (2001). Social competence. Gale Encyclopedia of Psychology. Disponível em http:// www.findarticles.com.

Carvalho, A., \& Guimarães, M. (2002). Desenvolvimento da criança de 0 a 6 anos: natureza e cultura em interação. Em A. Carvalho, F. Salles, \& M. Guimarães Desenvolvimento e Aprendizagem (Orgs). (pp. 31-50). Belo Horizonte: Editora UFMG.

Cury, C., \& Golfeto, J. (2002). Questionário de capacidades e dificuldades (SDQ): um estudo em escolares de Ribeirão Preto. Temas em Neurologia e Psiquiatria Infantil: uma visão multiprofissional. São Paulo: ABENEPI.

Del Prette, Z. A. P., \& Del Prette, A. (1999). Psicologia das Habilidades Sociais. Petrópolis: Vozes.

Del Prette, Z..A. P., \& Del Prette, A. (2001). Habilidades sociais e educação: Pesquisa e atuacão em psicologia escolar/ educacional. Em Z.A.P. del Prette (Org.). Psicologia Escolar e Educacional. (pp.99-128). Campinas: Alínea. número de participantes é muito pequeno para que sejam permitidas generalizações. Além disto, o número reduzido de participantes pode ter influenciado de forma significativa os resultados obtidos.

Division of Psychiatry-School of Community Health Sciences (2000). Research Activities in Developmental Psychiatry. Disponível: http://www.nottingham.ac.uk/psychiatry/ division/devpsych.htm. Acessado em 10/2001.

Dumas, D., \& Pelletier, A. (1999). A study of self perception in hyperactive children. American Journal of maternal/ Child Nursing, 24, 12-19.

Fleitlich, B., Cortázar, P.G., \& Goodman, R. (2000). Questionário de Capacidades e Dificuldades (SDQ). Infanto - Revista de Neuropsiquiatria da Infância e Adolescência,_8, 44-50.

Fontana, D. (1991). Psicologia para professores. São Paulo: Manole.

Garcia, C.P., Mazaira, J.A., \& Goodman, R. (2000). Validación inicial de la version gallega del Cuestionário de Capacidades y Dificultades (SDQ)/The initial validation study of the gallego version of the Strengths and Difficulties Questinnaire (SDQ). Revista de Psiquiatria Infanto Juvenil, 2, 95-100.

Goodman, R. (1997). The Strenghts and Difficulties Questionnaire: A Research Note. Journal of Child Psychology and Psychiatry, 38, 581-586.

Goodman, R., Meltzer, H., \& Bailey, V. (1998). The strengths and difficulties questionnaire: A pilot study on the validity of the self-report version. European Child and Adolescent Psychiatry, 7, 125-130.

Goodman, R., \& Scott, S. (1999). Comparing the Strengths and Difficulties Questionnaire and the Child Bahavior Checklist: Is small beautiful? Journal of Abnormal Child Psychology, 27, 17-24.

Goodman, R. (1999a). Questionário de Capacidades e Dificuldades (SDQ) Disponível: http://www. sdqinfo.com. Acessado em 15/05/2001.

Goodman, R. (1999). The Extended Version of the Strenghts and Difficulties Questionnaire as a Guide to Child Psychiatric Caseness and Consequent Burden. Journal of Child Psychology and Psychiatry, 40, 791-799.

Goodman, R., Renfrew, D., \& Mullick, M. (2000). Predicting type of psychiatric from Strengths and Difficulties Quetionnaire (SDQ) scores in child mental health clinics in London and Dhaka. European Child and Adolescent Psychiatry, 9, 129-134. 
Goodman, R., Ford, T., Simmons, H., Gatward, R., \& Meltzer, H. (2000). Using the Strengths and Difficulties Questionnaire (SDQ) to screen for child psychiatric disorders in a community sample. British Journal of Psychiatry, 177, 534-539.

Goodman, R. (2001). Psychometric Properties of the Strengths and Difficulties Quetionnaire. Journal of the American Academy of Child and Adolescent Psychiatry, 40, 13371345.

Grunspun, H. (1999). Crianças e Adolescentes com Transtornos Psicológicos e do Desenvolvimento. Rio de Janeiro: Atheneu.

Hartup, W.W. (1996). The company they keep: Friendships and their developmental significance. Child Development, 67, 1-13.

Hepper, F., \& Garralda, M.E. (2001). Psychiatric adjustment to leaving school in adolescents with intellectual disability: A pilot study. Journal of Intellectual Disability Research, 45, 521-525.

Klasen, H., Woerner, W., Wolke, D., Meyer, R., Overmeyer, S., Kaschnitz, W., Rothenberger, A., \& Goodman, R. (2000). Comparing the German versions of the Strenghts and Difficulties Questionnaire (SDQ-Deu) and the Child Behavior Checklist. European Child and Adolescent Psychiatry, 9, 271-276.

Kern, L. (2001) Improving the clsssroom behavior of students with emocional and behavioral disorders using individualized curricular modifications. Journal of Emotional and behavioral Disorders, 7, 234-245.

Koskelainen, M., Sourander, A., \& Vauras, M. (2001). Selfreported strengths and difficulties in a community sample of Finnish adolescents. European Child and Adolescent Psychiatry, 10(3), 180-185. [Abstract ].

Ladd, G.W., \& Hart, C.H. (1992). Creating informal play opportunities: Are parents and preschooler's initiations related to children's competence with peers? Developmental Psychology, 28, 1179-1187.

Ladd, G.W., Kochenderfer, B.J., \& Coleman, C.C. (1996). Friendship quality as a predictor of young children's early school adjustment. Child Development, 67, 1103-1118.

Ladd, G.W. (1999). Peer relationships and social competence during early and middle childhood. Annual Review of Psychology, 50, 333-359.

Lewin, L.M., Davis, B., \& Hops, H. (1999). Childhood Social Predictors of Adolescent Antisocial Behavior: Gender Differences in Predictive Accuracy and Efficacy. Journal of Abnormal Child Psychology, 56, 234-251.

Lindsay, G., \& Dockrell, J. (2000). The behavior and self-esteem of children with specific speech and language difficulties. British Journal of Educational Psychology, 70, 583-601.
Marcelli, D. (1998). Manual de Psicopatologia da Infância de Ajuriaguerra. Porto Alegre: ARTMED.

Mathai, J., Anderson, P., \& Bourne, A. (2002). The Strengths and Difficulties Questionnaire (SDQ) as a screening measure prior to admission to a child and adolescent Mental Health Service (CMHS). Australian e-journal for the advancement of

Mental Health, 1(3), 45-58.

Mcevoy, A. (2000). Antisocial bahavior, academic failure, and school climete: a critical review. Journal of Emotional and Behavioral Disorders, 20, 35-47.

McMunn, A.M., Nazroo, J.Y., Marmot, M.G., Boreham, R., \& Goodman, R. (2001). Children's emotional and behavioural well-being and the family environment: findings from the Health Survey for England. Society of Scientific Medicine 53, 423-40.

Merrell, K.W. (2001). An investigation of relationships between social behavior and ADHD in children and youth: construct validity of the home and community social behavior scales. Journal of Emotional and Behavioral Disorders. 52, 564-587.

Miller-Johnson, S., Coie, J.D., Maumary-Gremaud, A. e Bierman, K. (2002). Peer rejection and agression and early starter models of conduct disorder. Journal of Abnormal Child Psychology, 54, 345-356.

Newcombe, N. (1999). Desenvolvimento Infantil: Abordagem de Mussen. Porto Alegre: ARTMED.

Rohde, L.A., \& Benczik, E.B.P. (1999). Atenção/ Hiperatividade: O que é? Como ajudar? Porto Alegre: ARTMED.

Saud, L.F., \& Tonelotto, J.M.F. (2002). Autopercepção de Comportamentos Sociais no Ambiente Escolar. Pôster apresentado no Congresso da ABENEPI - Temas de Neurologia e Psiquiatria Infantil: uma visão multiprofissional. Curitiba, Pr.

Smedje, H., Broman, J.E., Hetta, J., \& VonKnorring, A.L. (1999). Psychometric properties of a swedish version of the "Strengths and Difficulties Questionnaire". European Child and Adolescent Psychiatry, 8(2), 63-70.

Smedje, H., Broman, J.E., \& Hetta, J. (2001). Associations between disturbed sleep and behavioural difficulties in 635 children aged six to eight years: A study based on parent's perceptions. European Child and Adolescent Psychiatry, 10, 1-9.

Thabet, A.A., Stretch, D., \& Vostanis, P. (2000). Child mental health problems in Arab children: Application of the Strengths and Difficulties Questionnaire. International Journal of Social Psychiatry, 46, 266-280. 
Topczewski, A. (1999). Hiperatividade. Como Lidar? São Paulo: Casa do Psicólogo.

Trad, L.A.B. (1999). Problematizando concepções de família e processos de interação social no contexto do Programa de Saúde da Família - PSF. Interfaces, 2, 103-106.

Vostanis, P., Tischler, V., Cumella, S., \& Bellerby, T. (2001). Mental health problems and social supports among homeless mothers and children victims of domestic and community violence. International Journal of Social Psychiatry, 47(4), 30-40.
Wentzel, K.R., \& Caldwell, K. (1997). Friendships, peer acceptance, and group membership: relations to academic in middle school. Child Development, 68, 1198-1209.

Recebido em: 04/05/05

Revisado em: 08/06/05

Aprovado em: 14/06/05

\section{Endereço para correspondência:}

Laura Fogaça Saud: Av. Granadeiro Guimarães, nº 46 - Centro - CEP: 12020-130 - Taubaté - SP

Josiane Maria de Freitas Tonelotto: Rua Antonio Prado, 116, apto. 202 - Centro - CEP: 13900-374 - Amparo - SP e-mail: jtonelotto@uol.com.br 